OPEN ACCESS

Edited by:

Ana Olivera,

National Institutes of Health (NIH),

United States

Reviewed by:

Manolo Sambucci,

Santa Lucia Foundation (IRCCS), Italy

Alex Dent,

Purdue University Indianapolis, United States

*Correspondence:

Kathryn A. Knoop

Knoop.Kathryn@mayo.edu

Rodney D. Newberry

rnewberry@wustl.edu

${ }^{+}$ORCID:

Phillip I. Tarr

orcid.org/0000-0003-4078-7517

Specialty section:

This article was submitted to

T Cell Biology,

a section of the journal

Frontiers in Immunology

Received: 09 September 2020 Accepted: 18 December 2020 Published: 03 February 2021

Citation:

Knoop KA, McDonald KG, Hsieh C-S, Tarr Pl and Newberry RD (2021) Regulatory T Cells Developing PeriWeaning Are Continually Required to Restrain Th2 Systemic

Responses Later in Life.

Front. Immunol. 11:603059. doi: 10.3389/fimmu.2020.603059

\section{Regulatory T Cells Developing Peri-Weaning Are Continually Required to Restrain Th2 Systemic Responses Later in Life}

\author{
Kathryn A. Knoop ${ }^{1,2 *}$, Keely G. McDonald ${ }^{1}$, Chyi-Song Hsieh ${ }^{1}$, Phillip I. Tarr ${ }^{1,3+}$ \\ and Rodney D. Newberry ${ }^{1 *}$
}

${ }^{1}$ Department of Internal Medicine, Washington University School of Medicine, St. Louis, MO, United States, ${ }^{2}$ Department of Immunology, Mayo Clinic, Rochester, MN, United States, ${ }^{3}$ Department of Pediatrics and Molecular Medicine, Washington University School of Medicine, St. Louis, MO, United States

Atopic disorders including allergic rhinitis, asthma, food allergy, and dermatitis, are increasingly prevalent in Western societies. These disorders are largely characterized by Thelper type 2 (Th2) immune responses to environmental triggers, particularly inhaled and dietary allergens. Exposure to such stimuli during early childhood reduces the frequency of allergies in at-risk children. These allergic responses can be restrained by regulatory $T$ cells (Tregs), particularly Tregs arising in the gut. The unique attributes of how early life exposure to diet and microbes shape the intestinal Treg population is a topic of significant interest. While imprinting during early life promotes the development of a balanced immune system and protects against immunopathology, it remains unclear if Tregs that develop in early life continue to restrain systemic inflammatory responses throughout adulthood. Here, an inducible deletion strategy was used to label Tregs at specified time points with a targeted mechanism to be deleted later. Deletion of the Tregs labeled peri-weaning at day of life 24, but not before weaning at day of life 14, resulted in increased circulating lgE and IL-13, and abrogated induction of tolerance towards new antigens. Thus, Tregs developing periweaning, but not before day of life 14 are continually required to restrain allergic responses into adulthood.

Keywords: regulatory $\mathrm{T}$ cells, ROR $\gamma \mathrm{T}$, Foxp3, weaning, allergy

\section{INTRODUCTION}

The prevalence of food allergies, perennial rhinitis, asthma, and eczema have increased dramatically in recent decades, especially among children $(1-3)$. These allergies are partly attributed to the loss or absence of tolerance to environmental antigens, a process largely meditated by Tregs, which express the transcription factor FoxP3. Current focus has turned to how tolerance induced in early life protects from the development of allergic disorders. Children at high risk for allergic disorders exposed to food allergens, such as peanut or egg, between 4 and 12 months of age had reduced risk 
of food sensitization compared to children avoiding allergens until after 5 years of age (4-7). Along with food allergen avoidance in early life, another risk factor for allergic disorders is antibiotic use in the first year of life, implicating a role for exposure to microbes in early life, and particularly microbes in the gastrointestinal tract, as protecting against allergy (8-10).

Within the spectrum of allergic disorders, IgE-mediated food allergy is particularly concerning due to its increasing incidence and life-threatening anaphylactic response on allergen consumption (11). Such IgE driven immune responses can be initiated and promoted by type 2 helper (Th2) responses, including the hallmark cytokine IL-13, which is predominantly produce by Th2 $\mathrm{T}$ cells, and promotes the production of IgE (12). Such responses can be suppressed by Tregs, therefore understanding the role of Tregs in pathogenesis of food allergy is a key to understanding how prevention of allergic disorders is best maintained.

Exposure to dietary allergens in the first year of life offers protection from future food allergies, while allergen avoidance until after five years of age is linked with increased food allergies (5-7, 13-15). These epidemiologic data are consistent with oral tolerance, or suppression of systemic responses to antigens and allergens first encountered in the gastrointestinal tract (16), having unique features when induced in early life (17). Induction of oral tolerance is correlated to the initiation of regulatory $\mathrm{T}$ cells that can suppress Th2 responses (18-20).

A population of Tregs expressing the transcription factor ROR $\gamma t+$ differentiate early in life in a process driven by the microbiota, and may have unique capacities to avoid immunopathologies and restrain Th2 responses $(21,22)$. Intriguingly this population of Tregs are reduced in children with food allergies (23). Children with food allergies had distinct microbiotas from healthy children, which induced significantly less ROR $\gamma t+$ Tregs (23) suggesting specific microbiota cues during early life are necessary for the development of this population of Tregs. Specific deletion of all Foxp3+ Tregs developing in early life increased gut inflammation, though the phenotype of the Tregs depleted was not determined (24). Moreover, reduced exposure to luminal antigens in early life decreased development of ROR $\gamma t+$ peripheral Tregs (pTregs) and was associated with an increase in Th2 responses to oral antigens (17). Adoptive transfer of ROR $\gamma t+p$ Tregs from isolated from the peri-weaning colon reduced Th2 responses against oral antigens suggesting early life ROR $\gamma t+$ pTregs are sufficient to restrain Th2 responses in an unbalanced immune system. Tregs promote tolerance through production of cytokines necessary for the tolerant environment, and therefore Tregs developing in early could contribute to the tolerogenic milieu that gives rise to future Tregs control Th2 responses. However we do not know if these Tregs are continually required to restrain Th2 responses later in life or if their role is restricted to early life to promote the development of a balanced immune system and once developed they are dispensable.

Here we show that the continued presence of "peri-weaning Tregs", Tregs developing prior to weaning, are necessary for the maintenance and development of tolerance to antigens encountered later in life. We labeled Tregs at weaning with a diphtheria toxin receptor to specifically delete adults of Tregs of early life origin which reduced ROR $\gamma t+$ pTregs, increased serum IgE and IL13, and abrogated tolerance to new orally administered antigens. Thus Tregs developing peri-weaning are a major source of ROR $\gamma \mathrm{t}+\mathrm{p}$ Tregs and are continually required to restrain Th2 responses in later life.

\section{MATERIALS AND METHODS}

\section{Mice}

All mice were maintained on the C57BL/6 background. C57BL/6 mice, OTII T-cell receptor transgenic mice (25), Foxp3 3 GPCreERT2 mice (26), RosalslDTR (27), were purchased from The Jackson Laboratory (Bar Harbor, ME). All mice were fed a routine chow diet. Co-housed littermates were used for experimental controls. All mice were weaned at DOL 21. Adult mice were 8 to 16 weeks of age when analyzed unless stated otherwise. Foxp3 3 GFCreERT2 mice and Rosa ${ }^{\text {lslDTR }}$ mice were bred for Foxp $3^{\text {GFPiDTR }}$ mice, which were injected with $100 \mu$ g tamoxifen (Sigma-Aldrich, St. Louis, MO, USA) dissolved in sunflower seed oil with $20 \%$ ethanol (Sigma-Aldrich) intraperitoneally (i.p.) on DOL 14 or 24. Mice were then aged to 8 weeks old (DOL 56), and injected with $50 \mu \mathrm{g} / \mathrm{kg}$ diphtheria toxin (DT) (Sigma Aldrich) i.p. In some experiments to validate deletion mechanism, Foxp $3^{\text {GFPiDTR }}$ mice were injected with tamoxifen on DOL21, and diphtheria toxin (DT) on DOL24. For controls, C57Bl/6 mice were injected with tamoxifen or vehicle (sunflower seed oil with $20 \%$ ethanol) on DOL24, and DT on DOL56 to minimize variation due to treatments. Animal procedures and protocols were performed in accordance with the Institutional Animal Care and Use Committee at Washington University School of Medicine.

\section{Isolation of Cellular Populations and Flow Cytometry}

Colons were harvested, rinsed with PBS, and colonic patches were removed. Isolation of splenic and LP cellular populations was performed as previously described (28). Colonic Treg subpopulations were identified as $7 \mathrm{AAD}^{-}, \mathrm{CD}^{+} 5^{+}, \mathrm{CD}^{+}$, $\mathrm{CD}^{+}$, Foxp $3^{+}$, and ROR $\gamma \mathrm{t}^{+}$. Foxp3 was identified using GFP signal. To detect intracellular antigens (ROR $\gamma$ t, cMAF, GATA3, and Tbet) and cytokines (IL13), cells were fixed and permeabilized overnight and stained per the manufacturer's recommendations (eBioscience). Flow cytometry was performed with a FACScan cytometer (BD Biosciences, San Jose, CA) retrofitted with additional lasers, or an Attune NXT four-laser flow cytometer (Invitrogen). Data acquisition and analysis were performed using Attune NXT software and FlowJo software (Tree Star, Ashland, OR). viSNE analysis was performed with FlowJo software.

\section{Oral Tolerance and Delayed Type Hypersensitivity Responses}

Mice were given Ova $20 \mathrm{~g} / \mathrm{L}$ in drinking water for 10 days beginning at DOL 70. Mice were then immunized subcutaneously with $100 \mu \mathrm{g}$ Ova in incomplete Freund's 
Adjuvant (Sigma Aldrich) 14 and 28 days later (i.e., DOL 84 and 98). At 16 weeks of age (DOL 112), mice were challenged with 20 $\mu \mathrm{g}$ Ova in the footpad and $24 \mathrm{~h}$ later the DTH response was read as the increase in footpad thickness before and after challenge as measured with micrometer calipers. Body temperature was monitored for one hour following challenge.

\section{Adoptive T-Cell Transfer of Ova-Specific T Cells}

Two days after the start of dietary Ova, mice were injected i.p. with $5 \times 10^{5}$ naïve Ova-specific $\mathrm{T}$ cells $\left(\mathrm{CD} 45.1^{+} \mathrm{CD} 3^{+} \mathrm{CD} 4^{+}\right.$ $\mathrm{CD}_{2} \mathrm{~L}^{+} \mathrm{V} \alpha 2^{+}$) fluorescence-activated cell sorting (FACS)isolated from spleens of adult OTII mice on the congenic CD45.1 background. Following transfer, organs were harvested at the indicated time points for cell isolation and analyzed by flow cytometry to detect and evaluate the phenotype of the CD $45.1^{+}$ OTII T-cells. Mice were evaluated seven days after transfer for the phenotype of the transferred CD45.1 $1^{+}$OTII T-cells.

\section{Measurement of Cytokines, and Immunoglobulins}

ELISAs specific for IL-13 (eBioscience), Eotaxin (R\&D systems), and IgE (eBiosciences) were used per manufacturers recommendations.

\section{Statistical Analysis}

Data were assumed to be normally distributed. Analysis was performed using a Student's $t$ test, one-way ANOVA with a Dunnett's post-test, or two-way ANOVA with a with GraphPad Prism (GraphPad Software Inc., San Diego, CA) and a two-tailed cutoff of $\mathrm{P}<0.05$ for significance.

\section{Study Approval}

Animal procedures and protocols were performed in accordance with the Institutional Animal Care and Use Committee at Washington University School of Medicine.

\section{RESULTS}

\section{RORyt+ Tregs Developing Peri-Weaning Persist Into Adulthood}

The induction of ROR $\gamma \mathrm{t}+$ pTregs begins before weaning and requires exposure to luminal antigens and microbes for their development. We have shown disruption of delivery of luminal substances to the lamina propria during early life reduced development of RORyt+ pTregs (17). This reduction remained statistically significant months later, and was associated with decreased oral tolerance induction and an increase in allergic-type responses against dietary antigens (17). We first asked if specific deletion of FoxP3 cells developing in early life was also associated with decreased oral tolerance. Mice expressing the diphtheria toxin receptor (DTR) exclusively in FoxP3+ cells (Foxp3 ${ }^{\text {DTR }}$ ) were injected with the diphtheria toxin (DT) on day of life (DOL) 14 or DOL24 (Figure 1A). FoxP3+ cells were significantly decreased on DOL28, in both the DT14 and DT24 groups (Figure 1B), but the FoxP3 compartment was restored by DOL120 (Figure 1B). Interestingly ROR $\gamma t+$ pTregs remained significantly decreased following DT-depletion of Foxp3 cells at DOL 24, but not at DOL 14 (Figure 1B). Thus ROR $\gamma t+$ pTregs develop after DOL 14 , in agreement with previous findings $(21,24,29)$.
A

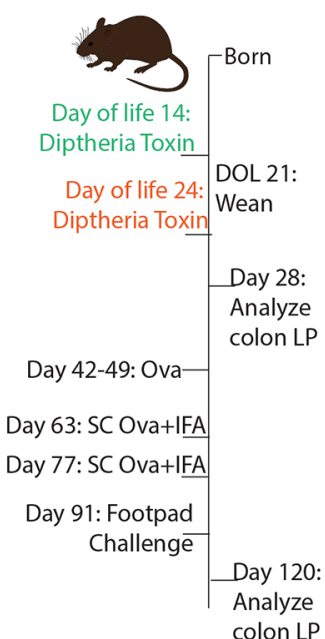

B

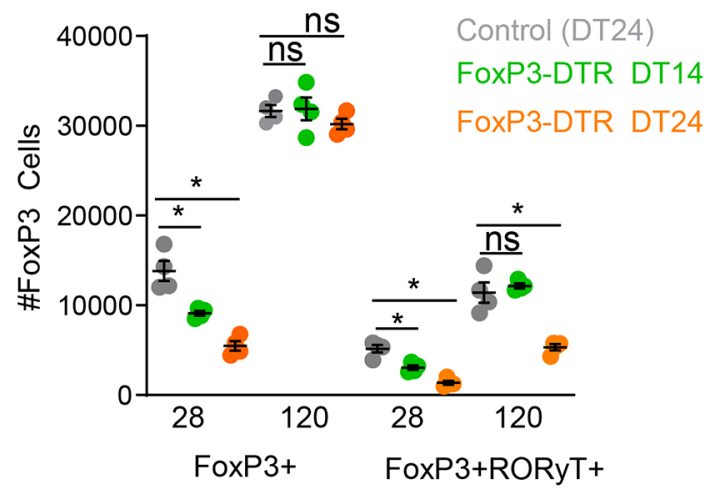

C

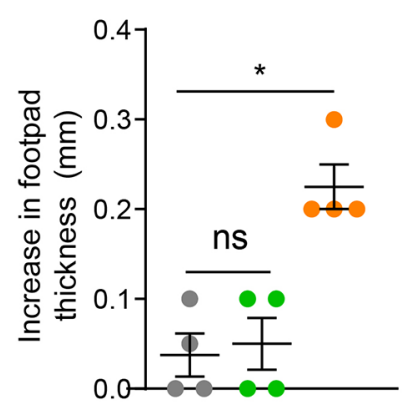

FIGURE 1 | Deletion of Tregs on DOL24, but not DOL14, abrogates oral tolerance later in life. (A) Schematic, FoxP3-DTR mice were injected with diphtheria toxin on DOL14 (green) or DOL24 (orange), control mice were injected with diphtheria toxin on DOL24 (gray). (B) Absolute number of Foxp3+ or RORyt+FoxP3+ colon LP cells analyzed on DOL 28 or DOL120. (C) Increase in footpad thickness following 7 days of Ova in drinking water, immunization, and footpad challenge with Ova, analyzed by one-way ANOVA with Dunnett's test for multiple comparison. $\mathrm{n}=4$ mice per group in panels $\mathrm{b}$ and $\mathrm{c}$, ${ }^{\star}$ denotes statistical significance $<0.05$, ns, not significant. 
Following deletion of Tregs in early life, we utilized an oral tolerance and delayed-hypersensitivity challenge model by exposing mice to ovalbumin (Ova) in drinking water, immunizing mice with Ova, challenging mice with Ova in the footpad, and measuring footpad swelling as an index of lack of tolerance. Mice had significantly increased footpad swelling, indicating reduced oral tolerance systemically, following deletion of Tregs at DOL24, but not DOL14. Taken along with our previous studies (17), these data highlight the importance of antigen delivery and development of Tregs during early life, but do not address if the ROR $\gamma t+$ pTregs arising during early life are continually required for constraining Th2 responses in later life or if once a balanced immune system is developed these Tregs are dispensable.

Mice expressing the diphtheria toxin receptor (DTR) under the tamoxifen driven cre in Foxp3 expressing cells, (Foxp ${ }^{\mathrm{iDTR}}$ ) were used to label Tregs arising at specific times in life to be deleted later. This construct permits the development of a balanced and healthy immune system, including the full Treg compartment and deletion of the Tregs developing in early life to assess their continuing role in later life. For initial validation of this system, Foxp $3^{\mathrm{iDTR}}$ and control mice were injected with tamoxifen on DOL21 for labeling with the DTR, which was followed by an injection with DT on DOL24 for deletion of labeled cells. A significant depletion in Foxp3+ cells was quantified on DOL28, indicating DTR was successfully expressed on Foxp3+ cells following tamoxifen labeling in the Foxp $3^{\text {iDTR }}$ and that DTR induced deletion of this population (Figure 2A), without significantly affecting other $\mathrm{T}$ cell subsets (Figure 2B).

Next, Foxp $3^{\text {iDTR }}$ mice were injected with tamoxifen on DOL 14 or 24, resulting in expression of the DTR in Foxp3+ cells present at those respective points. DT injection on DOL 56 depleted the Tregs that expressed Foxp3 on either DOL 14 or DOL 24 while leaving the Tregs developing after this time unchanged (Figure 2C). Depletion of early life Foxp3+ cells in adults resulted in no significant decrease in the total Foxp3+ population in the colon, as the labeled population of Tregs was a
A

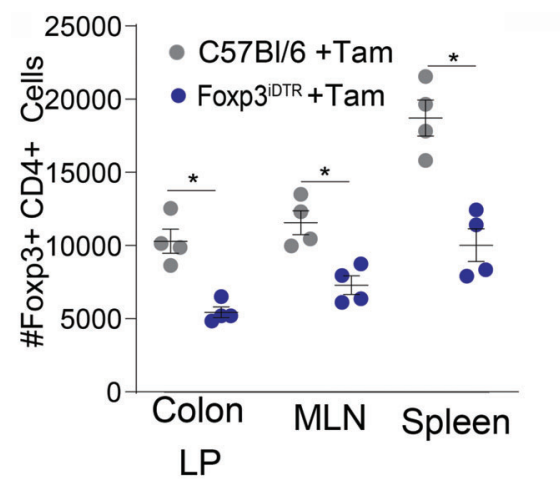

C

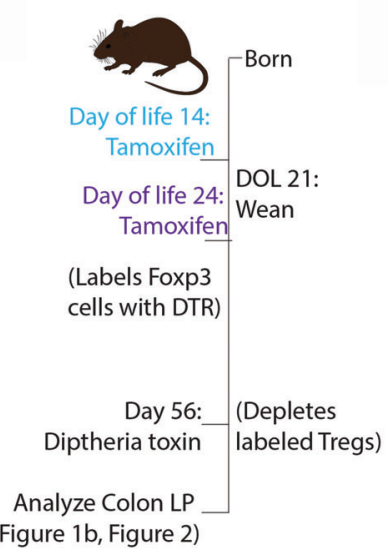

B
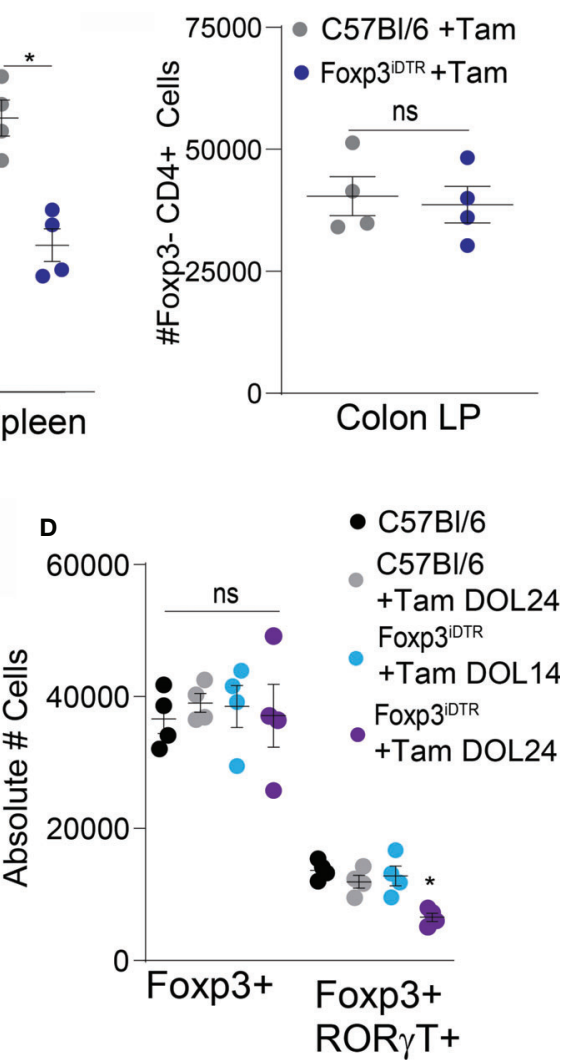

FIGURE 2 | ROR $\gamma t+F o x P 3+$ Tregs are induced peri-weaning and are present in the adult colon lamina propria. Control mice (gray) and FoxP3iDTR mice (dark blue) were injected with tamoxifen on DOL21, diphtheria toxin on DOL24, and analyzed on DOL28: (A) absolute number of Foxp3+ CD4 T cells and (B) absolute number of T cells subsets in colon lamina propria, analyzed by two-tailed Student's t test. (C) Experimental schematic describing labeling of Tregs with DTR using tamoxifen by injecting FoxP3iDTR mice on DOL14 (light blue) or DOL24 (purple). C57Bl6 mice were used as controls and injected with a vehicle (black) or tamoxifen on DOL24 (gray). All groups were injected with diphtheria toxin on DOL56 for deletion of labeled cells on DOL 56. (D) Absolute number of Foxp3+ or ROR $7 t+F o x P 3+$ colon LP cells analyzed on DOL 63 following depletion at DOL 56, analyzed by one-way ANOVA with Dunnett's test for multiple comparisons. $n=4$ mice per group in panels (A-C) *denotes statistical significance $<0.05$, ns, not significant. 
small proportion of the total Treg population at DOL 56 (Figure 2D). However, there was a significant decrease in the number of ROR $\gamma t+$ pTregs following DT-depletion of Foxp3 cells developing before DOL 24, but not before DOL 14 (Figure 2D). Confirming the critical time for ROR $\gamma t+$ pTregs development is after DOL $14(21,24,29)$, and in our colony largely do not develop after DOL 24 . We term all of the FoxP3+ cells developing between DOL14 and DOL24 "peri-weaning Tregs", which does include the ROR $\gamma t+$ pTregs (17). Tregs developing prior to DOL 14 are predominantly natural thymic derived Tregs and supress autoimmune responses later in life (30).

Multiparameter flow cytometry was performed on the CD4+ $\mathrm{T}$ cell population within the colon lamina propria following depletion of Tregs developing prior to DOL14 (Tam DOL14) or peri-weaning Tregs (Tam DOL24) in adults to evaluate the expression of the transcription factors Foxp3, ROR $\gamma$ t, cMaf, GATA3, and Tbet. Flow cytometry data was analyzed using a visualization tool for high-dimensional single-cell data based on the $t$-Distributed Stochastic Neighbor Embedding (t-SNE) algorithm, (viSNE), to visualize cell clusters and loss of cellular populations after deletion. No noticeable cluster was missing from following depletion of Tregs developing prior to DOL14, suggesting such cells either do not cluster into a distinct population, are not long-lived, or that a population with a similar pattern of transcription factor expression is also generated after DOL 14 (Figure 2). Depletion of peri-weaning Tregs, those labeled at DOL 24 and depleted in adults, greatly reduced the ROR $\gamma \mathrm{t}+\mathrm{Foxp} 3+$ cluster that included GATA3, cMaf, and Tbet expression (Figure 3). Taken together, these data suggest ROR $\gamma t+$ pTregs are largely generated in early life in mice in our colony, that these cell persist into adulthood, and that the peri-weaning Tregs includes a heterogenous population of Tregs expressing diverse transcription factors.

\section{Peri-Weaning Tregs Restrain Th2 Responses Later in Life}

To determine if peri-weaning Tregs were continually required to restrain Th2 responses, mice were monitored following depletion of early life Tregs. Serum IgE and IL13 concentrations were significantly elevated following depletion of peri-weaning Tregs, but not Tregs developing prior to DOL14 (Figures 4A, B). Serum IL-13 concentrations initially spiked following depletion of periweaning Tregs, but remained significantly elevated throughout the monitoring period. Serum IgE concentrations continued to increase suggesting peri-weaning Tregs are necessary for restraining systemic Th2 cytokines and antibodies.

Failure to develop the colonic Treg population in early life spontaneously skewed Th2 profiles that persisted into adulthood and impaired oral tolerance to dietary antigens initially encountered in adulthood. We assessed whether deletion of Tregs developing prior to DOL14 or peri-weaning Tregs in adults impaired oral tolerance to dietary antigens introduced in adulthood by deleting these Tregs in adult mice, exposing mice to ovalbumin (Ova) in drinking water, immunizing mice with Ova, challenging mice with Ova in the footpad, and measuring footpad swelling as an index of lack of tolerance (Figure 5A). Mice lacking peri-weaning Tregs had significantly increased footpad swelling, indicating reduced oral tolerance (Figure 5B). Additionally, Ova injected into the footpad of mice lacking peri-weaning Tregs caused a decrease in body temperature, suggestive of a hypersensitivity response to the injected antigen (Figure 5C).

Ova-specific CD4+ OTII T cells were transferred into mice receiving Ova in drinking water and analysed one week later, to phenotype antigen-specific T cell responses to dietary antigens in mice lacking Tregs developing prior to DOL14 or peri-weaning Tregs. A majority of the OTII T cells became Foxp3+ in the unmanipulated mice and mice depleted of Tregs developing prior to DOL14. In contrast, mice depleted of peri-weaning Tregs had significantly more OTII T cells with reduced Foxp3 expression and increased IL13 expression, suggesting an expansion of effector $\mathrm{T}$ cells specific for dietary Ova in the absence of peri-weaning Tregs and a portion of these expressed Th2 cytokines (Figure 5D).

\section{DISCUSSION}

Our results demonstrate that continued presence of peri-weaning + Tregs, including ROR $\gamma t+$ pTregs, in adulthood is necessary to restrain Th2 responses and tolerance to dietary antigens encountered initially in adulthood. We have shown that ROR $\gamma t+$ pTregs developing in the colon are longer-lived in the absence of cognate antigen (17). Integrating these observations with prior studies suggests that there are periods in life for the development of Tregs with distinct properties. The first period occurs in the first week of life in mice during which naturally derived Tregs specific for self-antigens develop which function to protect from autoimmunity (30). The next period spanning the interval between the first week through weaning in mice is defined the formation of goblet cell-associated antigen passages (GAPs) in the colon. During this time peripherally induced Tregs (pTregs) specific for non-self-antigens develop extrathymically, a mechanism of Treg induction important for restraint of Th2 responses (31). A portion of these pTregs express the transcription factor ROR $\gamma t$ and their development is driven by the microbiota, which is potentially delivered to the colon via GAPs $(17,32)$. The ROR $\gamma t+$ pTregs developing during this period are longer-lived than those developing in adulthood and have the capacity to restrain Th2 responses and promote tolerance to new antigens throughout life. While ROR $\gamma t+$ pTregs can develop throughout life, our data suggest that the ROR $\gamma t+$ pTregs developing after weaning are insufficient to restrain immunopathology.

Intriguingly, peri-weaning Tregs, which includes ROR $\gamma \mathrm{t}+$ pTregs, restrained future responses in our model in a nonantigen specific manner suggesting here Tregs act in an innate manner to shape and promote a tolerogenic environment. Within the context of food allergy, the reduction or absence of ROR $\gamma t+$ pTregs could shift the immune environment, influencing both effector subsets and innate cells, within the intestine, allowing for pathogenesis of allergic reactions. 

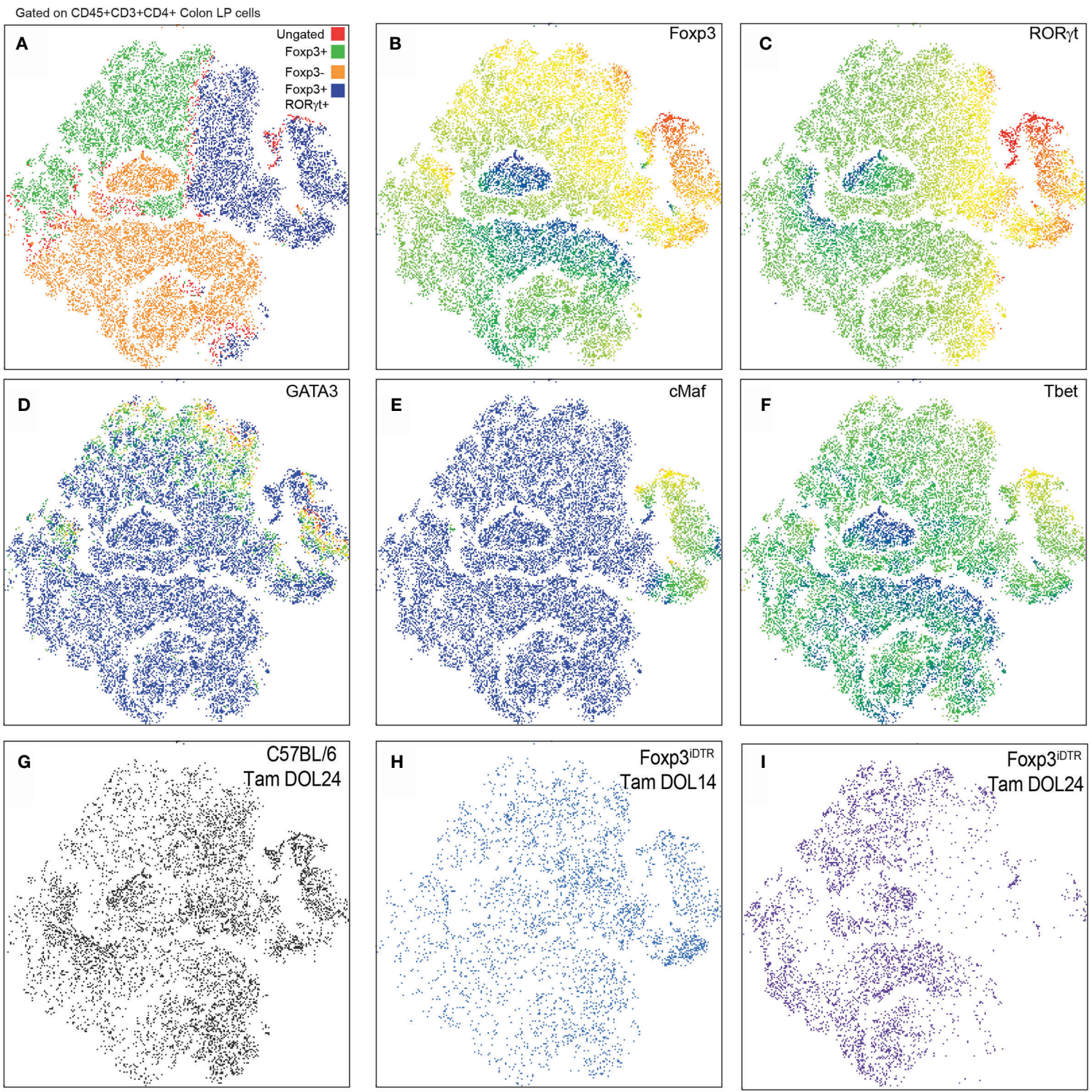

FIGURE 3 | RORYt+FoxP3+ peri-weaning Tregs include a heterogeneous population of Tregs that express GATA3, CMAF, and Tbet. (A) viSNE plots of colon CD4 T cell populations from pooled samples denoting Foxp3+ and RORYt+Foxp3+ and Foxp3- clusters. (B-F) Plots also show individual expression of Foxp3, RORt, GATA3, cMAF, and Tbet. (G-I) Plots of CD4+ T cells in the colon LP from individual experimental conditions. Vsne analysis in (A-F) are pooled from $n=3$ mice per group, plots in (G-I) are representative of $\mathrm{n}=3$ mice per group.

Following depletion of peri-weaning Tregs, IgE steadily increased suggesting IgE-producing plasma cell populations either expand or increase secretion. The role of IgE in food allergy is well described, and we previously showed an association of decreased ROR $\gamma t+$ pTregs to increased allergen-specific $\operatorname{IgE}(17,32)$. However, here it remains to be seen if increased total IgE may contribute to future allergic sensitization (33) or just indicate a skewing of the immune system to favor allergic responses. Similarly, increased serum IL13 suggests expansion or increased activity of Th2 effector cells or innate cells such as eosinophils and basophils. IL13 has been shown to not only drive goblet cell hyperplasia, but also increase allergen delivery across the intestinal epithelium by the formation of GAPs and secretory cell associated passages (SAPs) (34), and been implemented in promoting oral allergen sensitization (35) and food allergy pathogenesis (33). Thus increased systemic IL13 has the potential to increase delivery of food allergens into the lamina propria and sensitize innate cells triggering the pathogenesis of allergic responses. How peri-weaning Tregs restrain IL13 production and secretion through either direct cell contact or indirectly is of great interest. While more data is necessary to understand how ROR $\gamma t+$ pTregs uniquely prevent allergic reactions, it remains clear their development during early life may be imperative to maintaining tolerance throughout life. 

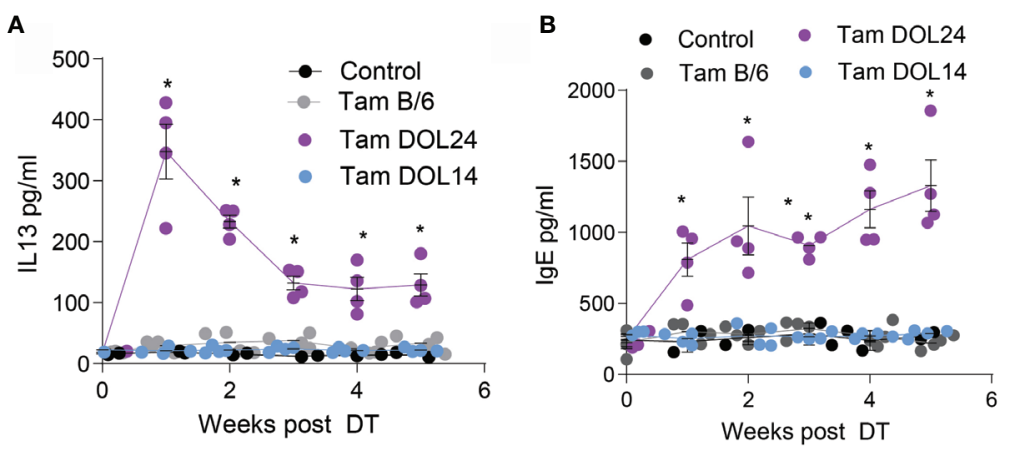

FIGURE 4 | Systemic IL13 and IgE is increased following depletion of peri-weaning Tregs. Serum concentrations of (A) IL13 or (B) total IgE for 5 weeks following deletion of Tregs. $n=4$ mice per group in panels (A, B), analyzed by two-way ANOVA with Dunnett's test for multiple comparisons. * denotes statistical significance $<0.05$.

A

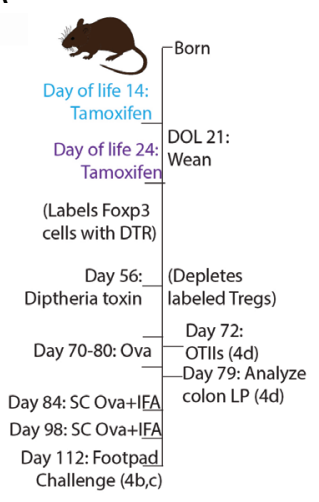

C

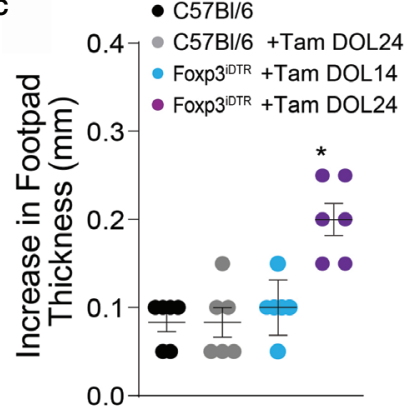

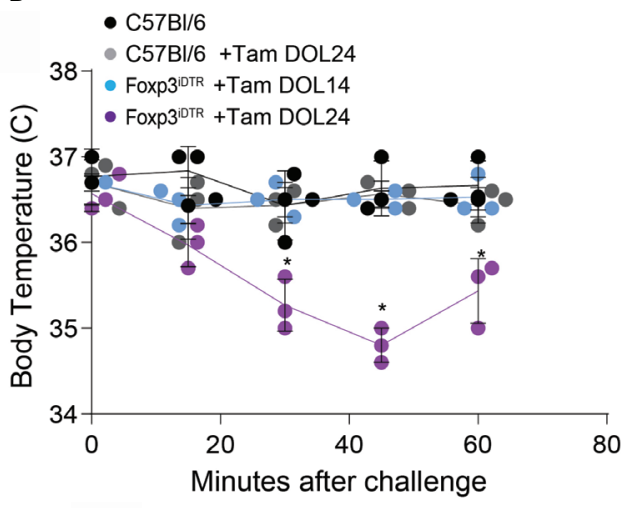

D

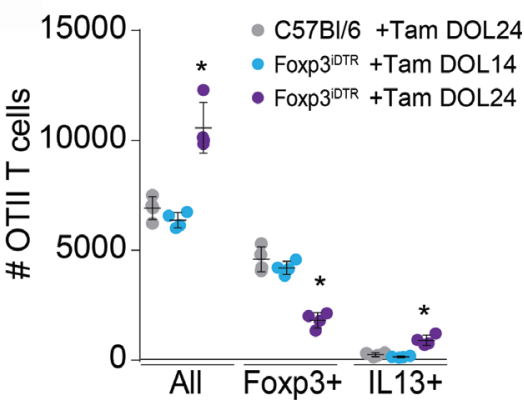

FIGURE 5 | Development of tolerance to new oral antigens is abrogated following depletion of peri-weaning Tregs. (A) Experimental schematic describing labeling of Tregs with DTR using tamoxifen followed by deletion of labeled cells on DOL 56, and DTH response (left, B and C) or transfer of OTII cells (right, D) FoxP3iDTR mice were injected with tamoxifen on DOL14 (light blue) or DOL24 (purple). C57BI6 mice were used as controls and injected with a vehicle (black) or tamoxifen on DOL24 (gray). All groups were injected with diphtheria toxin on DOL56 for deletion of labeled cells on DOL 56. (B) Increase in footpad thickness following 7 days of Ova in drinking water, immunization, and footpad challenge with Ova, analyzed by one-way ANOVA with Dunnett's test for multiple comparison. (C) Change in temperature following footpad challenge, analyzed by two-way ANOVA with Tukey's test for multiple comparisons. (D) Number of Ova-specific OTII cells in the colon LP 7 days following adoptive transfer and dietary Ova, analyzed by one-way ANOVA with Dunnett's test for multiple comparison. $\mathrm{n}=6$ mice per group in panels (B, C) from two independent experiments, c displays data from one representative experiment, $n=3$ mice in panel (D) * denotes statistical significance $<0.05$. 
While the generation and function of these pTregs is an area of active investigation, multiple features, that may be unique to early life, have been identified to contribute to the development of this ROR $\gamma t+$ pTreg population. There are two key time points for the role of antigen delivery: GAP formation in the colon prior to weaning, and GAP and SAP formation in the small intestine later in life. The former drives ROR $\gamma \mathrm{t}+\mathrm{pTregs}$ expansion in response to microbial products, while the later has the potential to promote food allergy pathogenesis through increased food allergen delivery (34). Understanding how the antigen delivery process results in two very different outcomes depends on both location (colon as compared to small intestine) and timing (peri-weaning compared to later in life). During the pre-weaning interval in which naïve $\mathrm{T}$ cells are stimulated to generate these pTregs, dietary and microbial antigens are delivered to the colon lamina propria via GAPs to generate antigen-specific immune responses $(17,32)$. This process is regulated by maternal ligands present in breastmilk and bacterial components of the maturing microbiota around weaning, most notably epidermal growth factor $(17,32)$. Maternal antibodies IgA and IgG also present in breastmilk have been shown to promote tolerance, may protect from food allergy pathogenesis in early life, and may help regulate development of ROR $\gamma t+$ pTregs $(22,36,37)$. Following weaning, dietary antigens are largely encountered by the immune system in the small intestine, and dietary antigen-specific pTregs developing post-weaning have a limited life span in absence of antigen exposure $(38,39)$. This mechanism might explain why allergen avoidance in early life does not reduce food allergy. Indeed, complementary diets combined food allergens with breastmilk protect against food allergy later in life (14). These feeding practices could be directing food allergens to the colon during the first year of life to promote long lived tolerance via the induction of dietary antigen-specific ROR $\gamma t+$ pTregs.

Another feature supporting the development of this ROR $\gamma \mathrm{t}+$ pTreg population is the gut microbiota. The requirement of the microbiota for inducing ROR $\gamma t+p$ Tregs has long been appreciated $(21,29)$. Antibiotic use, particularly in the first year of life, is strongly associated with later life food allergies (40), and would certainly induce microbial dysbiosis (41). Children with food allergies have distinct microbiotas from healthy children. These bacterial communities induced significantly fewer ROR $\gamma t+$ pTregs (23). Additionally Th2 responses have the potential to create feedback loops, depleting the bacterial taxa necessary for Treg development (42). Administration of individual or a consortium of bacteria partly rescued ROR $\gamma t+$ pTregs where such cells were otherwise lacking or decreased (23). However this effect was restricted to specific bacterial taxa, suggesting microbial cues exclusive to the early life microbiota promote the development of this population of pTregs $(43,44)$. However attempts to manipulate the microbiota as a therapy for allergies have had limited success possibly due to the lack of other unique features present in early life.

Finally, ROR $\gamma t+$ pTregs are likely different depending on the time of life during which the pTregs differentiate. Recently a set point for the ROR $\gamma t+$ pTreg population was described to be defined in early life, shortly after birth (22), and pTregs may have age-dependent fates (45). ROR $\gamma \mathrm{t}+$ expression can be driven by bacteria adherent to the intestinal epithelium in adult mice, including in pTregs (46-48), however pTregs induced in this manner are transient and dependent on the continued presence of the microbiota (29). The manner in which adherent bacterial antigens are encountered by the immune system post-weaning differs from how commensal bacterial antigens are encountered pre-weaning $(32,49)$. Common adherent bacteria have not been observed in the peri-weaning microbiota $(50,51)$, suggesting other microbial members or luminal factors drive ROR $\gamma \mathrm{t}$ expression peri-weaning. During differentiation ROR $\gamma \mathrm{t}+$ pTregs potentially express Foxp3 first, becoming a Foxp3+ ROR $\gamma t$ - intermediate before final differentiation into ROR $\gamma t$ + Foxp3+ (52). Intriguingly induction of Foxp3 and ROR $\gamma \mathrm{t}$ expression may be initiated by differential microbial products $(53,54)$, potentially sensed directly by the Tregs $(23)$.

Taken together, peri-weaning pTregs including the ROR $\gamma \mathrm{t}+$ pTregs subset induced in the colon, are uniquely capable of enduring suppression systemic inflammation later in life. The heterogenous expression of specific transcription factors suggest peri-weaning Tregs can restrain multiple Th-driven inflammatory responses (55-57). One limitation of this study is through the labeling of all Foxp3 expressing cells at a specified time in life, deletion of cells upon diphtheria treatment results in mass deletion of Foxp 3 cells present at the specified labeling time point, suggesting the mass deletion of cells from a specified time point may be as important as their specificity or gene expression pattern representative of the Foxp3 population.

It remains unclear how peri-weaning ROR $\gamma t+$ pTregs restrain allergic responses uniquely when compared to other Tregs subsets. Multiple described roles for Tregs in the restraint of allergic responses include producing cytokines that suppress immune responses, modulating antigen presenting cells to prevent antigen presentation, and preventing proliferation of $\mathrm{T}$ effector cells (58-60). While our data are consistent with each of these functions and suggest peri-weaning Tregs are continually required to perform their suppressive role, one alternative interpretation is the presence of the $\mathrm{ROR} \gamma \mathrm{t}+$ peri-weaning pTregs limits or decreases differentiation of future Tregs capable of restraining Th2 responses through active utilization of space and resources in the lamina propria as these are longlived Tregs (17). Future work should explore if the peri-weaning Treg phenotype can be replicated later in life.

In conclusion, we have shown the continued presence of periweaning Tregs is necessary to restrain Th2 responses and supports tolerance to dietary antigens encountered later in life. These results implicate peri-weaning Tregs, which include a substantial population of ROR $\gamma t+$ pTregs, as playing active roles in suppressing potential Th2 responses and maintaining tolerogenic homeostasis. Further these findings underscore the importance of early life immune education for the proper expansion of this population, as Tregs developing post-weaning do not substitute for the peri-weaning Tregs. These data suggest the peri-weaning ROR $\gamma \mathrm{t}+$ pTregs have unique capacities and are potentially not replaced without intervention. Thus risk factors for food allergy such as antibiotic use and allergen avoidance may 
contribute to food sensitization by disrupting this population of peri-weaning pTregs.

\section{DATA AVAILABILITY STATEMENT}

The original contributions presented in the study are included in the article/supplementary material. Further inquiries can be directed to the corresponding authors.

\section{ETHICS STATEMENT}

The animal study was reviewed and approved by Institutional Animal Care and Use Committee at Washington University School of Medicine.

\section{REFERENCES}

1. Branum AM, Lukacs SL. Food allergy among U.S. children: trends in prevalence and hospitalizations. NCHS Data Brief (2008) 10:1-8.

2. Jackson KD, Howie LD, Akinbami LJ. Trends in allergic conditions among children: United States, 1997-2011. NCHS Data Brief (2013) 121:1-8.

3. Gupta RS, Warren CM, Smith BM, Blumenstock JA, Jiang J, Davis MM, et al. The Public Health Impact of Parent-Reported Childhood Food Allergies in the United States. Pediatrics (2018) 142:e20181235. doi: 10.1542/peds.2018-1235

4. Poole JA, Barriga K, Leung DY, Hoffman M, Eisenbarth GS, Rewers M, et al. Timing of initial exposure to cereal grains and the risk of wheat allergy. Pediatrics (2006) 117:2175-82. doi: 10.1542/peds.2005-1803

5. Nwaru BI, Erkkola M, Ahonen S, Kaila M, Haapala AM, Kronberg-Kippila C, et al. Age at the introduction of solid foods during the first year and allergic sensitization at age 5 years. Pediatrics (2010) 125:50-9. doi: 10.1542/peds.2009-0813

6. Koplin JJ, Osborne NJ, Wake M, Martin PE, Gurrin LC, Robinson MN, et al. Can early introduction of egg prevent egg allergy in infants? A population-based study. J Allergy Clin Immunol (2010) 126:807-13. doi: 10.1016/j.jaci.2010.07.028

7. Du Toit G, Roberts G, Sayre PH, Bahnson HT, Radulovic S, Santos AF, et al. Randomized Trial of Peanut Consumption in Infants at Risk for Peanut Allergy. New Engl J Med (2015) 372:803-13. doi: 10.1056/NEJMoa1414850

8. Metsala J, Lundqvist A, Virta LJ, Kaila M, Gissler M, Virtanen SM. Mother's and offspring's use of antibiotics and infant allergy to cow's milk. Epidemiology (2013) 24:303-9. doi: 10.1097/EDE.0b013e31827f520f

9. Love BL, Mann JR, Hardin JW, Lu ZK, Cox C, Amrol DJ. Antibiotic prescription and food allergy in young children. Allergy Asthma Clin Immunol Off J Can Soc Allergy Clin Immunol (2016) 12:41. doi: 10.1186/ s13223-016-0148-7

10. Ni J, Friedman H, Boyd BC, Mcgurn A, Babinski P, Markossian T, et al. Early antibiotic exposure and development of asthma and allergic rhinitis in childhood. BMC Pediatr (2019) 19:225. doi: 10.1186/s12887-019-1594-4

11. Anvari S, Miller J, Yeh CY, Davis CM. IgE-Mediated Food Allergy. Clin Rev Allergy Immunol (2019) 57:244-60. doi: 10.1007/s12016-018-8710-3

12. De Vries JE. The role of IL-13 and its receptor in allergy and inflammatory responses. J Allergy Clin Immunol (1998) 102:165-9. doi: 10.1016/S0091-6749 (98)70080-6

13. Koplin JJ, Dharmage SC, Ponsonby AL, Tang ML, Lowe AJ, Gurrin LC, et al. Environmental and demographic risk factors for egg allergy in a populationbased study of infants. Allergy (2012) 67:1415-22. doi: 10.1111/all.12015

14. Du Toit G, Sayre PH, Roberts G, Sever ML, Lawson K, Bahnson HT, et al. Effect of Avoidance on Peanut Allergy after Early Peanut Consumption. N Engl J Med (2016) 374:1435-43. doi: 10.1056/NEJMoa1514209

15. Du Toit G, Katz Y, Sasieni P, Mesher D, Maleki SJ, Fisher HR, et al. Early consumption of peanuts in infancy is associated with a low prevalence of peanut allergy. J Allergy Clin Immunol (2008) 122:984-91. doi: 10.1016/j.jaci.2008.08.039

\section{AUTHOR CONTRIBUTIONS}

KK performed sample collection, ELISAs, flow cytometry, tSNE analysis, and data analysis. KK, and $\mathrm{KM}$ performed animal breeding, genotyping, and injections. KK, C-SH, PT, and RN designed the experiments, analyzed and interpreted the data, and wrote the manuscript. All authors contributed to the article and approved the submitted version.

\section{FUNDING}

Supported by grants: DK052574-PIT and RDN, DK097317RDN, AI131342-RDN, AI1407551-RDN and CSH, AI136515RDN and CSH, AI112626-SPH and RDN, AI131349-CSH, DK109006-KAK, and DK122187-KAK.

16. Pabst O, Mowat AM. Oral tolerance to food protein. Mucosal Immunol (2012) 5:232. doi: 10.1038/mi.2012.4

17. Knoop KA, Mcdonald KG, Coughlin PE, Kulkarni DH, Gustafsson JK, Rusconi B, et al. Synchronization of mothers and offspring promotes tolerance and limits allergy. JCI Insight (2020) 5. doi: 10.1172/jci.insight.137943

18. Chehade M, Mayer L. Oral tolerance and its relation to food hypersensitivities. J Allergy Clin Immunol (2005) 115:3-12. doi: 10.1016/j.jaci.2004.11.008

19. Wawrzyniak M, O'mahony L, Akdis M. Role of Regulatory Cells in Oral Tolerance. Allergy Asthma Immunol Res (2017) 9:107-15. doi: 10.4168/ aair.2017.9.2.107

20. Tordesillas L, Berin MC. Mechanisms of Oral Tolerance. Clin Rev Allergy Immunol (2018) 55:107-17. doi: 10.1007/s12016-018-8680-5

21. Ohnmacht C, Park J-H, Cording S, Wing JB, Atarashi K, Obata Y, et al. The microbiota regulates type 2 immunity through ROR $\gamma t+$ T cells. Science (2015) 349:989-93. doi: 10.1126/science.aac4263

22. Ramanan D, Sefik E, Galván-Peña S, Wu M, Yang L, Yang Z, et al. An Immunologic Mode of Multigenerational Transmission Governs a Gut Treg Setpoint. Cell (2020) 181:1276-90.e1213. doi: 10.1016/j.cell.2020.04.030

23. Abdel-Gadir A, Stephen-Victor E, Gerber GK, Noval Rivas M, Wang S, Harb H, et al. Microbiota therapy acts via a regulatory T cell MyD88/ROR $\gamma t$ pathway to suppress food allergy. Nat Med (2019) 25:1164-74. doi: 10.1038/s41591-0190461-z

24. Al Nabhani Z, Dulauroy S, Marques R, Cousu C, Al Bounny S, Déjardin F, et al. A Weaning Reaction to Microbiota Is Required for Resistance to Immunopathologies in the Adult. Immunity (2019) 50:1276-88.e1275. doi: 10.1016/j.immuni.2019.02.014

25. Barnden MJ, Allison J, Heath WR, Carbone FR. Defective TCR expression in transgenic mice constructed using cDNA-based alpha- and beta-chain genes under the control of heterologous regulatory elements. Immunol Cell Biol (1998) 76:34-40. doi: 10.1046/j.1440-1711.1998.00709.x

26. Rubtsov YP, Niec RE, Josefowicz S, Li L, Darce J, Mathis D, et al. Stability of the regulatory T cell lineage in vivo. Science (2010) 329:1667-71. doi: 10.1126/ science.1191996

27. Buch T, Heppner FL, Tertilt C, Heinen T, Kremer M, Wunderlich FT, et al. A Cre-inducible diphtheria toxin receptor mediates cell lineage ablation after toxin administration. Nat Methods (2005) 2:419. doi: 10.1038/ nmeth762

28. Mcdonald KG, Leach MR, Brooke KW, Wang C, Wheeler LW, Hanly EK, et al. Epithelial expression of the cytosolic retinoid chaperone cellular retinol binding protein II is essential for in vivo imprinting of local gut dendritic cells by lumenal retinoids. Am J Pathol (2012) 180:984-97. doi: 10.1016/ j.ajpath.2011.11.009

29. Sefik E, Geva-Zatorsky N, Oh S, Konnikova L, Zemmour D, Mcguire AM, et al. Individual intestinal symbionts induce a distinct population of ROR $\gamma+$ regulatory T cells. Science (2015) 349:993-7. doi: 10.1126/science.aaa9420 
30. Yang S, Fujikado N, Kolodin D, Benoist C, Mathis D. Immune tolerance. Regulatory $\mathrm{T}$ cells generated early in life play a distinct role in maintaining self-tolerance. Science (2015) 348:589-94. doi: 10.1126/science.aaa7017

31. Josefowicz SZ, Niec RE, Kim HY, Treuting P, Chinen T, Zheng Y, et al. Extrathymically generated regulatory $\mathrm{T}$ cells control mucosal TH2 inflammation. Nature (2012) 482:395-9. doi: 10.1038/nature10772

32. Knoop KA, Gustafsson JK, Mcdonald KG, Kulkarni DH, Coughlin PE, Mccrate S, et al. Microbial antigen encounter during a preweaning interval is critical for tolerance to gut bacteria. Sci Immunol (2017) 2. doi: 10.1126/ sciimmunol.aao1314

33. Wang M, Takeda K, Shiraishi Y, Okamoto M, Dakhama A, Joetham A, et al. Peanut-induced intestinal allergy is mediated through a mast cell-IgEFcepsilonRI-IL-13 pathway. J Allergy Clin Immunol (2010) 126:306-316, 316.e301-312. doi: 10.1016/j.jaci.2010.05.017

34. Noah TK, Knoop KA, Mcdonald KG, Gustafsson JK, Waggoner L, Vanoni S, et al. IL-13-induced intestinal secretory epithelial cell antigen passages are required for IgE-mediated food-induced anaphylaxis. J Allergy Clin Immunol (2019) 144:1058-73.e1053. doi: 10.1016/j.jaci.2019.04.030

35. Brandt EB, Munitz A, Orekov T, Mingler MK, Mcbride M, Finkelman FD, et al. Targeting IL-4/IL-13 signaling to alleviate oral allergen-induced diarrhea. J Allergy Clin Immunol (2009) 123:53-8. doi: 10.1016/j.jaci.2008.10.001

36. Mosconi E, Rekima A, Seitz-Polski B, Kanda A, Fleury S, Tissandie E, et al. Breast milk immune complexes are potent inducers of oral tolerance in neonates and prevent asthma development. Mucosal Immunol (2010) 3:461-74. doi: 10.1038/mi.2010.23

37. Ohsaki A, Venturelli N, Buccigrosso TM, Osganian SK, Lee J, Blumberg RS, et al. Maternal IgG immune complexes induce food allergen-specific tolerance in offspring. J Exp Med (2018) 215:91-113. doi: 10.1084/jem.20171163

38. Kim KS, Hong S-W, Han D, Yi J, Jung J, Yang B-G, et al. Dietary antigens limit mucosal immunity by inducing regulatory $\mathrm{T}$ cells in the small intestine. Science (2016) 351:858-63. doi: 10.1126/science.aac5560

39. Kulkarni DH, Gustafsson JK, Knoop KA, Mcdonald KG, Bidani SS, Davis JE, et al. Goblet cell associated antigen passages support the induction and maintenance of oral tolerance. Mucosal Immunol (2020) 13:271-82. doi: 10.1038/s41385-019-0240-7

40. Netea SA, Messina NL, Curtis N. Early-life antibiotic exposure and childhood food allergy: A systematic review. J Allergy Clin Immunol (2019) 144:1445-8. doi: 10.1016/j.jaci.2019.08.001

41. Shu SA, Yuen AWT, Woo E, Chu KH, Kwan HS, Yang GX, et al. Microbiota and Food Allergy. Clin Rev Allergy Immunol (2019) 57:83-97. doi: 10.1007/ s12016-018-8723-y

42. Campbell C, Dikiy S, Bhattarai SK, Chinen T, Matheis F, Calafiore M, et al. Extrathymically Generated Regulatory T Cells Establish a Niche for Intestinal Border-Dwelling Bacteria and Affect Physiologic Metabolite Balance. Immunity (2018) 48:1245-57.e1249. doi: 10.1016/j.immuni.2018.04.013

43. Stefka AT, Feehley T, Tripathi P, Qiu J, Mccoy K, Mazmanian SK, et al. Commensal bacteria protect against food allergen sensitization. Proc Natl Acad Sci U.S.A. (2014) 111:13145-50. doi: 10.1073/pnas.1412008111

44. Feehley T, Plunkett CH, Bao R, Choi Hong SM, Culleen E, Belda-Ferre P, et al. Healthy infants harbor intestinal bacteria that protect against food allergy. Nat Med (2019) 25:448-53. doi: 10.1038/s41591-018-0324-z

45. Pratama A, Schnell A, Mathis D, Benoist C. Developmental and cellular age direct conversion of CD4+ T cells into ROR $\gamma+$ or Helios+ colon Treg cells. J Exp Med (2019) 217. doi: 10.1084/jem.20190428

46. Ivanov II, Atarashi K, Manel N, Brodie EL, Shima T, Karaoz U, et al. Induction of Intestinal Th17 Cells by Segmented Filamentous Bacteria. Cell (2009) 139:485-98. doi: 10.1016/j.cell.2009.09.033

47. Lathrop SK, Bloom SM, Rao SM, Nutsch K, Lio CW, Santacruz N, et al. Peripheral education of the immune system by colonic commensal microbiota. Nature (2011) 478:250-4. doi: 10.1038/nature10434
48. Chai JN, Peng Y, Rengarajan S, Solomon BD, Ai TL, Shen Z, et al. Helicobacter species are potent drivers of colonic $\mathrm{T}$ cell responses in homeostasis and inflammation. Sci Immunol (2017) 2. doi: 10.1126/sciimmunol.aal5068

49. Ladinsky MS, Araujo LP, Zhang X, Veltri J, Galan-Diez M, Soualhi S, et al. Endocytosis of commensal antigens by intestinal epithelial cells regulates mucosal T cell homeostasis. Science (2019) 363:eaat4042. doi: 10.1126/ science.aat 4042

50. Ivanov II, Frutos RDL, Manel N, Yoshinaga K, Rifkin DB, Sartor RB, et al. Specific Microbiota Direct the Differentiation of IL-17-Producing T-Helper Cells in the Mucosa of the Small Intestine. Cell Host Microbe (2008) 4:337-49. doi: 10.1016/j.chom.2008.09.009

51. Nutsch K, Chai JN, Ai TL, Russler-Germain E, Feehley T, Nagler CR, et al. Rapid and Efficient Generation of Regulatory T Cells to Commensal Antigens in the Periphery. Cell Rep (2016) 17:206-20. doi: 10.1016/ j.celrep.2016.08.092

52. Solomon BD, Hsieh C-S. Antigen-Specific Development of Mucosal Foxp3 + ROR $\gamma t+$ T Cells from Regulatory T Cell Precursors. J Immunol (Baltimore Md 1950) (2016) 197:3512-9. doi: 10.4049/jimmunol.1601217

53. Atarashi K, Tanoue T, Shima T, Imaoka A, Kuwahara T, Momose Y, et al. Induction of colonic regulatory $\mathrm{T}$ cells by indigenous Clostridium species. Science (2011) 331:337-41. doi: 10.1126/science.1198469

54. Atarashi K, Tanoue T, Oshima K, Suda W, Nagano Y, Nishikawa H, et al. T induction by a rationally selected mixture of Clostridia strains from the human microbiota. Nature (2013) 500:232-6. doi: 10.1038/nature12331

55. Wang Y, Su MA, Wan YY. An essential role of the transcription factor GATA3 for the function of regulatory T cells. Immunity (2011) 35:337-48. doi: 10.1016/j.immuni.2011.08.012

56. Wohlfert EA, Grainger JR, Bouladoux N, Konkel JE, Oldenhove G, Ribeiro $\mathrm{CH}$, et al. GATA3 controls Foxp $3^{+}$regulatory T cell fate during inflammation in mice. J Clin Invest (2011) 121:4503-15. doi: 10.1172/JCI57456

57. Xu M, Pokrovskii M, Ding Y, Yi R, Au C, Harrison OJ, et al. c-MAFdependent regulatory $\mathrm{T}$ cells mediate immunological tolerance to a gut pathobiont. Nature (2018) 554:373-7. doi: 10.1038/nature25500

58. Noval Rivas M, Chatila TA. Regulatory T cells in allergic diseases. J Allergy Clin Immunol (2016) 138:639-52. doi: 10.1016/j.jaci.2016.06.003

59. Satitsuksanoa P, Jansen K, Głobińska A, Van De Veen W, Akdis M. Regulatory Immune Mechanisms in Tolerance to Food Allergy. Front Immunol (2018) 9:2939. doi: 10.3389/fimmu.2018.02939

60. Calzada D, Baos S, Cremades-Jimeno L, Cárdaba B. Immunological Mechanisms in Allergic Diseases and Allergen Tolerance: The Role of Treg Cells. J Immunol Res (2018) 2018:6012053-6012053. doi: 10.1155/2018/6012053

Conflict of Interest: RN, KK, and KM are inventors on U.S. Nonprovisional Application Serial No. 15/880,658 Compositions and Methods for Modulation of Dietary and Microbial Exposure. PT discloses a financial conflict of interest with MediBeacon Inc (member of their Scientific Advisory Board, consultant, and equity holder), is a consultant to Kallyope Inc., and is a potential recipient of royalties from a patent to test human gut permeability noninvasively.

The remaining author declares that the research was conducted in the absence of any commercial or financial relationships that could be construed as a potential conflict of interest.

Copyright (c) 2021 Knoop, McDonald, Hsieh, Tarr and Newberry. This is an open-access article distributed under the terms of the Creative Commons Attribution License (CC BY). The use, distribution or reproduction in other forums is permitted, provided the original author(s) and the copyright owner(s) are credited and that the original publication in this journal is cited, in accordance with accepted academic practice. No use, distribution or reproduction is permitted which does not comply with these terms. 